Revue d'histoire de l'Amérique française

REVUE D.HISTOIRE DE L'AMÉRIQUE FRANÇAISE

\title{
Les lieux historiques de la région de Québec comme lieux d'expression identitaire, 1965-1985
}

\section{Patrice Groulx et Alain Roy}

Volume 48, numéro 4, printemps 1995

URI : https://id.erudit.org/iderudit/305364ar

DOI : https://doi.org/10.7202/305364ar

Aller au sommaire du numéro

Éditeur(s)

Institut d'histoire de l'Amérique française

ISSN

0035-2357 (imprimé)

1492-1383 (numérique)

Découvrir la revue

Citer cette note

Groulx, P. \& Roy, A. (1995). Les lieux historiques de la région de Québec comme lieux d'expression identitaire, 1965-1985. Revue d'histoire de l'Amérique française, 48(4), 527-541. https://doi.org/10.7202/305364ar
Résumé de l'article

Au cours de la période 1965-1985, les lieux historiques pourvus de centres d'interprétation ou de musées se sont multipliés à Québec et dans sa région. Ce phénomène est l'expression d'une nouvelle formulation de l'identité québécoise dans la foulée de la Révolution tranquille. Il répond aussi aux visées stratégiques des gouvernements du Québec et du Canada dans leur recours à la mémoire collective. Les lieux historiques diffusent une vision du passé traversée par l'idée de modernité et par une redéfinition des appartenances et du patrimoine. Ils offrent un terrain d'étude fertile et ne doivent pas échapper au regard critique de la recherche historique. 


\title{
NOTE DE RECHERCHE
}

\section{LES LIEUX HISTORIQUES DE LA RÉGION DE QUÉBEC COMME LIEUX D'EXPRESSION IDENTITAIRE, 1965-1985'}

\author{
PATRICE GROULX \\ ALAIN ROY \\ Département d'histoire \\ Université Laval
}

\section{RÉSUMÉ}

Au cours de la période 1965-1985, les lieux historiques pourvus de centres d'interprétation ou de musées se sont multipliés à Québec et dans sa région. Ce phénomène est l'expression d'une nouvelle formulation de l'identité québécoise dans la foulée de la Révolution tranquilie. Il répond aussi aux visées stratégiques des gouvernements du Québec et du Canada dans leur recours à la mémoire collective. Les lieux historiques diffusent une vision du passé traversée par l'idée de modernité et par une redéfinition des aupartenances et du patrimoine. Ils offrent un terrain d'étude fertile et ne doivent pas échapper au regard critique de la recherche historique.

\section{ABSTRACT}

In the years 1965-1985, historic sites associated with interpretative centres or museums have multiplied in Quebec City and surrounding areas. This pkenomenon

1. Les auteurs remercient les évaluateurs de la Revue d'histoire de l'Amérique française pour leurs judicieux commentaires.

2. La présente note expose les conclusions provisoires d'une recherche dirigée par Jocelyn Létourneau, professeur d'histoire à l'Université Laval et membre du Centre d'études interdisciplinaires sur les Lettres, les Arts et les Traditions (CÉLAT). Elle fait partie d'un programme de recherche subventionné par le Conseil de recherches en sciences humaines du Canada et qui porte sur les «Usages de faits du passé et de la tradition dans la construction identitaire des Québécois: les années 1970». Les hypothèses générales sur lesquelles se fondent ce programme sont les suivantes: 1) les années 1960-1970 sont marquées par la n: issance symbolique d'un être québécois «moderne», en rupture avec un passé «traditionnel» et folklorisé; 2) cette rupture est associée à une ouverture du «Nous» envers l' «Autre» en même temps qu'à une volonté de réenracinement dans le passé; 3) ce nouvel enracinement suscite une reconfiguration de la mémoire, dans laquelle les lieux historiques jouent un rôle de premier plan. Ces hypothèses ont été explicitées dans plusieurs articles de Jocelyn Létourneau, et particulièrement dans «Le 'Québec moderne', un chapitre du grand récit collectif des Québécois», Revue française de science politique, 42,5 (octobre 1992): 765-785. 
reflects a new expression of Quebec's identity in the wake of the Quiet Revolution. It also reflects the strategic aims of the governments of Quebec and Canada in fostering the collective memory. The vision of the past disseminated by historic sites is supported by the idea of modernity and a redefinition of belonging and heritage. These sites are a stimulating object of study and should not escape the historian's critical gaze.

Au cours des années 1960 et par la suite, alors que le Québec vit ce qu'on appelle la Révolution tranquille, le discours sur la modernité et l'ouverture à l'Autre devient un élément important de l'expression collective. En même temps, les centres d'interprétation et les lieux et musées historiques se multiplient après 1965 . Éléments centraux d'une nouvelle formulation de la mémoire nationale, ces lieux sont des miroirs de l'identité et leur étude est révélatrice de la façon dont la société québécoise, par son actualisation du passé, se pense et se redéfinit ${ }^{3}$. Comme l'écrit Jean Hamelin,

les musées ne sont pas des institutions neutres, innocentes, qui évoluent en dehors des luttes socio-politiques. Ils sont davantage que des refuges pour les débris d'un passé révolu. Fenêtres ouvertes sur l'Autre et miroirs d'un Nous collectif, ils sont les cellules vivantes d'une mémoire sociale qui créent et recréent sans cesse un passé accordé à ce que nous croyons être ${ }^{4}$.

Pourtant, la représentation du passé offerte par ces lieux de production et de diffusion de mémoire a été longtemps négligée par les historiens. Depuis quelques années, cependant, de nombreux chercheurs abordent les lieux d'interprétation du patrimoine et les musées comme terrain d'étude sur les rapports entre histoire, mémoire

3. Le thème de la mémoire collective a fait l'objet de nombreuses recherches, qu'il serait trop long d'énumérer ici. Dans la production québécoise, voir, entre autres, Jacques Mathieu, dir., Étude de la construction de la mémoire collective des Québécois au XXe siècle (Québec, CÉLAT, 1986); et John Porter, dir., Recherches actuelles et mémoires collectives (Québec, CÉLAT, 1988). Par ailleurs, les chercheurs québécois commencent à s'intéresser aux rapports qui lient le récit historique et la production d'une identité collective, dont Jacques Mathieu et Jacques Lacoursière, Les mémoires québécoises (Sainte-Foy, Presses de l'Université Laval, 1992). La réflexion a surtout visé la production savante avec, par exemple, les travaux de Jean-Paul Bernard, Les rébellions de 1837-1838 (Montréal, Boréal Express, 1983). Voir aussi les travaux de Fernand Dumont et Fernand Harvey, «La recherche sur la culture», Recherches sociographiques, 26,1-2 (1985): 85-118; de Jacques Mathieu et al., Approches de l'identité québécoise (Québec, CÉLAT, 1985); et de Jocelyn Létourneau, «L'historiographie comme miroir, écho et récit de nous-autres», communication présentée à l'université Duke, septembre 1993.

4. Jean Hamelin, Le Musée du Québec: Histoire d'une institution nationale (Québec, Musée du Québec, 1991), 9-10. 
et identités. Dans cette perspective, nous avons tenté de cerner la contribution des lieux historiques à la reconstitution mémorielle et identitaire associée à la Révolution tranquille. Pour ce faire il s'agissait, dans un premier temps, de saisir les transformations dans le rapport collectif au passé exprimé par l'évolution de ces lieux de mémoire. Dans un deuxième temps, plutôt que de vérifier si l'histoire était «correctement» présentée, nous nous sommes attardés à cerner la représentation globale du passé que l'on tente de recréer ou de mettre en scène.

\section{I - MÉTHODOLOGIE}

Notre recherche repose sur une double démarche. Tout d'abord, une étude documentaire a permis de tracer les grandes lignes de l'intervention étatique dans le champ de la mémoire et de l'identité nationales. Parallèlement, nous avons confronté ces orientations au développement concret des lieux historiques. Nous avons donc procédé à la visite systématique des lieux historiques sélectionnés ${ }^{6}$, recueilli brochures et dépliants et effectué des entrevues.

Afin de réaliser cette analyse qualitative, nous avons déterminé un corpus homogène et représentatif. Nous avons d'abord défini, comme

5. Voir en particulier les travaux suivants: Béatrice Verge, «L'interprétation du patrimoine architectural: sites historiques et muséologie à Québec», mémoire de maitrise, Québec, Université Laval, 1985; Susan Porter Benson, Stephen Brier et Roy Rosenzweig, eds., Presenting the Past: Essays on History and the Public (Philadelphia, Temple University Press, 1986); Jo Blatti, ed., Past Meets Present (Washington, Smithsonian Institution Press, 1987); Warren Leon et Roy Rosenzweig, eds., History Museums in the United States: a Critical Assessment (Chicago, University of Illinois Press, 1989); Patrice Groulx, «Une mémoire momifiée? Problèmes et perspectives de l'interprétation de l'histoire dans les centres d'interprétation", mémoire de maîtrise, Montréal, Université du Québec à Montréal, 1990; Ivan Karp, Christine M. Kreamer et Steven D. Lavine, eds., Museums and Communities: the Politics of Public Culture (Washington, Smithsonian Institution Press, 1992); Thomas J. Schlereth, Cultural History and Material Culture: Everyday Life, Landscapes, Museums (Charlottesville, University Press of Virginia, 1992); Kevin Walsh, The Representation of the Past: Museums and Heritage in the Post-Modern World (Londres, Routledge, 1992); Cyril Simard, Andrée Lapointe et Corneliu Kirjan, Patrimoine muséologique au Québec: repères chronologiques (Québec, Commission des biens culturels, 1992); Andrée Lapointe, «L'incidence des politiques culturelles sur le développement des musées nationaux Canada-Québec depuis 1950», thèse de doctorat, Québec, Université Laval, 1993.

6. Pour ce faire, nous avons élaboré un questionnaire d'enquête et de visite. Il est intéressant de noter que, même si les expositions sont un phénomène important dans la diffusion d'un discours sur le passé, les outils pour leur examen critique par les historiens sont rares. Nous nous sommes inspirés de ceux proposés par Thomas Schlereth dans «Museum Exhibition Review. Introduction», Journal of American History, 76,1 (juin 1989): 192-195 et «American Material Culture Technique: Historical Museum Exhibit Review» dans Artefacts and the American Past (Nashville, Association for State and Local History, 1980). Lors de notre enquête à l'été 1993, nous n'avons considéré que les expositions permanentes et en visite libre. 
lieu historique, un lieu 1) chargé de mémoire, c'est-à-dire un lieu physique ancien; 2) en partie ou totalement restauré ou reconstruit; et 3 ) où le discours historique porte sur le lieu proprement dit. Cette définition, concentrée sur le lieu physique et le discours historique que l'on tient à son propos, élimine un certain nombre de musées où l'on met en valeur des collections et où l'on traite peu du lieu comme tel. Afin de dégager les tendances dans la représentation du passé tel qu'exprimé au cours des années 1970, nous n'avons considéré que les expositions présentées dans ces lieux entre 1965 et $1985^{7}$. Afin de rendre compte des nuances dans le traitement de la mémoire en fonction des types de propriétaires, nous avons étudié les divers lieux présents dans la grande région de Québec. Après avoir recensé tous les lieux où un discours sur le passé a été présenté durant la période cible, nous avons retenu 21 lieux qui correspondent à la définition visée, qui sont situés dans la région déterminée et qui ont ouvert ou existaient déjà entre 1965 et $1985^{8}$. Nous avons par la suite classé ces divers lieux en fonction de leur forme de propriété ou de gestion, et particulièrement en fonction du rôle de l'État' 9

7. C'est durant cette période qu'on observe l'essentiel des efforts de reconfiguration de la mémoire dans des lieux ouverts au public. Les projets de diffusion exigent souvent de longues étapes de préparation et des interventions sur des bâtiments patrimoniaux, sans compter les retards causés par les querelles de juridiction entre ministères et paliers de gouvernement. Cela explique que des lieux planifiés entre 1965 et 1970, par exemple, n'ouvrent au public que dans la décennie suivante.

8. Il faut bien comprendre que nous parlons de lieux historiques au sens large et non de musées au sens classique. Pour la recension, nous avons eu recours à la collection de guides touristiques du ministère du Tourisme du Québec (1969-1983) et aux guides des régions de Québec et de Chaudière-Appalaches publiés à partir de 1980, de même qu'à l'ouvrage de Guy Boulizon, Les musées du Québec (Montréal, Fides, 1976), 2 tomes. De la soixantaine de lieux répertoriés, nous avons retenu, à Québec même: 1) l'Hôpital général et son musée, 2) l'HôtelDieu et son musée, 3) le Musée des Ursulines, 4) La Citadelle et son musée, 5) la Place-Royale et ses centres d'interprétation, 6) le Centre d'interprétation du Vieux-Port, ainsi que les lieux historiques nationaux, 7) Cartier-Brébeuf, 8) Parc-de-l'Artillerie, 9) Fortifications-de-Québec; en périphérie et sur la rive Nord: 10) la Chapelle Notre-Dame-de-Lorette, et 11) la Maison Aroüanne (Wendake), 12) la Villa-Bagatelle (Sillery), 13) le Manoir Mauvide-Genest (SaintJean de l'île d'Orléans), 14) le Centre d'interprétation de la Côte-de-Beaupré (Château-Richer), 15) l'Historial (Sainte-Anne-de-Beaupré); sur la rive Sud: 16) le Domaine Joly de Lotbinière (Sainte-Croix-de-Lotbinière), 17) la Maison Alphonse-Desjardins, 18) le lieu historique national du Fort-Numéro-Un (Lévis-Lauzon), 19) le Moulin de Beaumont, 20) le Moulin Bernier (SaintAubert), et 21) la Seigneurie des Aulnaies (Saint-Roch-des-Aulnaies). Notons que parmi les sites retenus la plupart ont été financés à titre de centres d'animation patrimoniale, et non en vertu des politiques d'accréditation muséale. Nous n'avons pas pu étudier d'autres sites qui répondaient à nos critères de sélection, faute de documentation sur les expositions et l'état des lieux entre 1965 et 1985. En effet, les lieux historiques, avec l'exception notable de ceux de Parcs Canada, conservent très peu de traces sur leur nouvelle affectation en lieux de mémoire. On présente rarement au public la réutilisation d'un site comme élément de son histoire. D'autre part, on accorde peu d'intérêt à la préservation des documents qui vont permettre d'analyser $a$ posteriori les expositions présentées.

9. Nous avons ainsi retenu trois grandes catégories de lieux: A) les lieux publics, c'està-dire dont la propriété et la gestion sont totalement du domaine public (par exemple, Parcs 
Nous allons maintenant examiner le développement des lieux historiques en relation avec les politiques culturelles de l'État. Par la suite, nous aborderons divers aspects de la reconstruction identitaire qu'ils incarnent, c'est-à-dire la question de la modernité, la définition du rapport entre le Nous et l'Autre, et finalement l'articulation du rapport collectif au passé.

\section{2 - ÉVOLUTION DES LIEUX HISTORIQUES ET DES POLITIQUES CULTURELLES}

Dans la région de Québec, la mémoire consignée dans les lieux historiques au cours des années 1950-1960 est d'abord une mémoire d'institutions; elle vise une clientèle interne et rayonne peu. La préservation par l'État du patrimoine bâti s'adresse avant tout à un public cultivé et vise principalement à recréer une ambiance historique. L'ouverture en 1965 de la Maison Chevalier, à Place-Royale, est la première tentative notable de mise en valeur du patrimoine à l'intention du grand public. À la même époque, toutefois, l'État élabore déjà les grands projets qui verront le jour dans la décennie 1970.

La période 1970-1980 est marquée par l'intervention directe et massive des deux paliers supérieurs de l'État. On entreprend de reconstruire des ensembles urbains imposants: Place-Royale, sous la direction du gouvernement provincial mais avec l'injection de fonds fédéraux, Parc-de-l'Artillerie et Cartier-Brébeuf dans les propriétés fédérales. On recourt assez tôt à des formules populaires de mise en valeur, principalement des centres d'interprétation (Maison Fornel de Place-Royale et Cartier-Brébeuf en 1972, Parc-de-l'Artillerie en 1973), des activités d'animation et des visites guidées. Ces moyens de mise en valeur seront systématiquement employés jusqu'à aujourd'hui. La mémoire des lieux historiques, qui jusque-là misait sur la nostalgie ou le pittoresque, se transforme ainsi en objet de connaissance. Dans l'aménagement des lieux, on recourt à l'interprétation pour organiser le sens à donner au patrimoine, notamment avec des scénarios de visite et des textes explicatifs.

À partir de 1975 apparaissent des conflits. Jusque-là, les grands projets de reconstruction, qui modifient pourtant en profondeur le

Canada). Dans le corpus (voir la note précédente), ils portent les numéros 5, 6, 7, 8, 9, 14, 18; B) les lieux mixtes, dont la propriété, le financement ou la gestion sont à la fois publics et privés (généralement à la suite d'une entente entre un organisme sans but lucratif et l'État), et qui portent les numéros $10,11,12,16,21 ; C)$ les lieux privés au plan de la gestion et de la propriété mais ouverts au public (parfois, ces lieux reçoivent un financement d'appoint de l'État). Il s'agit des numéros 1, 2, 3, 4, 13,15, 17, 19 et 20. Parmi ces derniers, la plupart relèvent d'institutions religieuses, militaires ou corporatives. 
paysage patrimonial et la trame urbaine du Vieux-Québec, ne font pas l'objet d'interrogations marquées. Après quelques années apparaissent des résistances ou des tensions entre les communautés locales et le ministère des Affaires culturelles (MAC) dans le traitement des lieux historiques. Ainsi, l'objectif même du colloque Place-Royale, tenu en 1978 , est de résoudre des conflits de divers ordres.

En périphérie, ces tensions portent sur le caractère exemplaire des projets patrimoniaux: les critères d'authenticité que le MAC met de l'avant entrent en contradiction avec les attentes moins rigides qui animent les promoteurs locaux lorsqu'ils réclament une aide à la restauration. Des conflits apparaissent par exemple au Moulin du Petit-Pré (Centre d'interprétation de la Côte-de-Beaupré), au Domaine Joly de Lotbinière, à la Seigneurie des Aulnaies ou à la VillaBagatelle.

Ces tensions, qui naissent d'ailleurs dans un contexte de crise budgétaire, mènent à la différenciation des interventions provinciales et fédérales. Au palier provincial, le dirigisme brutal qui caractérise le début des années 1970 cède la place, au milieu de la décennie, à des partenariats. Cela ne va pas sans heurt, les ressources gouvernementales étant orientées vers les milieux qui lancent des initiatives. Par contraste, l'intervention fédérale est imposée dans des lieux prédéterminés et se pratique à sens unique.

Conséquence des initiatives locales, des conflits et des nouvelles approches du MAC, les lieux mixtes se multiplient à partir de 1975. La poussée est encore timide dans un premier temps, mais se confirme nettement à partir de 1980 . On remarque aussi que durant les années 1975-1980, les anciens lieux de mémoire institutionnels, c'est-à-dire la Citadelle de Québec et les musées des communautés religieuses, consacrent des efforts notables pour s'ouvrir au grand public.

La mise en valeur du patrimoine a toujours été considérée comme un élément de la vie culturelle et, à ce chapitre, l'État joue un rôle décisif. En ce qui concerne le gouvernement fédéral, son intervention directe dans les lieux historiques remonte au début du siècle, mais les orientations récentes prennent leur source dans les études et les conclusions de la commission Massey sur la culture canadienne, dont le rapport est publié en 1951.

Deux idées centrales animent les politiques culturelles fédérales. La première est celle du renforcement de l'identité canadienne face à l'envahissement de la culture américaine; la seconde, celle du renforcement de l'unité canadienne dans le creuset d'une culture commune, qui échapperait par nature aux tensions linguistiques et «régionales». Dès la commission Massey et dans toutes les décisions prises 
par la suite, on attribue un rôle de ciment identitaire au patrimoine. En effet, devant la constatation que le Canada, comme «nation», n'a pas de mythes ou de symboles fondateurs et reconnus de tous, on souhaite créer un sentiment d'appartenance par le biais de la diffusion publique d'un passé commun dans des lieux et des musées d'histoire.

L'intervention fédérale dans la mémoire est donc stratégique. Comme l'énonce la Politique des lieux historiques nationaux de 1968, elle doit être conçue comme structurante, soutenue par des mises de fonds considérables et hiérarchiser le patrimoine en lieux importants, sous juridiction fédérale, et secondaires, sous d'autres juridictions. Dans la foulée de cette politique, Parcs Canada, principal mandataire du gouvernement fédéral pour la gestion de la mémoire collective, produit au tournant des années 1980 un ambitieux Plan de réseau qui thématise et organise l'histoire du pays en vue de consolider les anciens lieux historiques et d'en établir de nouveaux ${ }^{10}$.

À Québec, dès le milieu des années 1950, le gouvernement fédéral prévoit intégrer divers sites dans son réseau national. À cet effet, il acquiert des terrains et des bâtiments (Fortifications, parc CartierBrébeuf, Maison Maillou, secteur de l'Arsenal), de sorte qu'au début des années 1960 les propriétés de Parcs Canada dépassent en importance celles de la Commission des champs de bataille nationaux, vénérable institution propriétaire des plaines d'Abraham depuis le début du siècle. Ce n'est qu'au cours des années 1970 que l'intervention fédérale s'amplifie. En 1972 et 1973, à Cartier-Brébeuf et au Parc-de-l'Artillerie apparaissent les premiers centres d'interprétation

10. Pour ce qui est des politiques culturelles fédérales touchant au patrimoine, voir notamment les documents suivants: Gouvernement du Canada, Rapport de la Commission royale d'enquête sur l'avancement des arts, lettres et sciences au Canada (Ottawa, Cloutier, 1951); Hilda Neatby, «National History», Recueil de quelques études spéciales. Commission royale d'enquête sur l'avancement des arts, des lettres et des sciences au Canada. 1949-1951 (Ottawa, Cloutier, 1951), 205-217; Charles Bilodeau, «L'histoire nationale», ibid., 217-231; W. L. Morton, «Historical Societies and Museums», ibid., 249-261; Ministère des Affaires Indiennes et du Nord canadien, Direction des parcs nationaux et des lieux historiques, Politique des lieux historiques nationaux (Ottawa, 1968); Groupe d'étude sur le Plan du réseau des parcs historiques nationaux, Le plan du réseau des parcs historiques nationaux, Étape 1, juillet-octobre 1979. Version définitive (Ottawa, 1979); id., Plan du réseau des parcs historiques nationaux, Étape 2, Première version (Ottawa, 1980); id., Plan du réseau des parcs historiques nationaux, Étape 3, Mise en auvre, Première version (Ottawa, 1981). Brooke Jeffrey, La politique culturelle du Canada de Massey-Lévesque à Appelbaum-Hébert (Ottawa, Bibliothèque du Parlement, décembre 1982); Parcs Canada, Politique de Parcs Canada (Ottawa, 1980), 75 p.; Comité d'étude de la politique culturelle fédérale, Rapport (Ottawa, Ministère des Communications, 1982). On trouvera des renseignements sur l'histoire des lieux historiques fédéraux dans les ouvrages suivants: W. F. Lothian, Petite histoire des parcs nationaux du Canada (Ottawa, Ministère des Approvisionnements et Services du Canada, 1987); C. J. Taylor, Negociating the Past: the Making of Canada's National Historic Parks and Sites (Montréal et Kingston, McGillQueen's University Press, 1990). 
de la région. D'autres équipements verront le jour jusqu'en 1984. Par ailleurs, le fédéral investira plusieurs millions de dollars dans PlaceRoyale et dans certains musées grâce à des ententes auxiliaires.

Du côté du gouvernement du Québec, le Rapport de la commission Tremblay, publié en 1956, affirme la nécessité d'une intervention soutenue de l'État dans le domaine culturel. Posant l'identité canadienne-française comme un fait acquis, la Commission affirme que cette intervention est devenue nécessaire pour édifier une culture commune qui permettra d'atténuer les contradictions sociales.

La création du ministère des Affaires culturelles en 1961 et le Livre blanc sur la culture de 1965 continuent sur cette lancée. Dans ce dernier document, l'État québécois est présenté comme l'incarnation politique de la nationalité canadienne-française et comme le mobilisateur des énergies collectives. La culture étant perçue comme un domaine où les possibilités d'action de la nation sont illimitées, l'intervention de l'État pour la diffuser devient une nécessité pressante.

En ce qui concerne le patrimoine, le gouvernement du Québec intervient par des dispositions législatives sur la protection des biens culturels en 1956 et 1963. Mais surtout, il met en branle l'ambitieux projet de Place-Royale en 1966, qui résulte de la problématique identitaire énoncée dans le Livre blanc de 1965.

Le début de la décennie 1970 est marqué par une nouvelle idée du patrimoine, qui se voit reconnaître comme bien culturel et s'élargit à l'ensemble de la mémoire tangible et intangible. La mission du patrimoine est alors de contribuer au développement de la culture et résulte notamment de pressions des milieux locaux, qui réclament une ouverture au pluralisme et à la diffusion. La Déclaration du ministre Hardy en 1974 et le Livre vert du ministre L'Allier en 1976 explicitent également une volonté d'affirmation nationale par rapport au gouvernement fédéral; on est bien à l'époque de la «souveraineté culturelle». Une stratégie se dessine pour contrecarrer la présence fédérale: recherche d'alliances avec les groupes du milieu, développement de l'idée d'un réseau de lieux historiques, et diversification des investissements dans leur acquisition et leur mise en valeur.

Le Livre blanc de 1978, publié peu après l'arrivée au pouvoir du Parti québécois, complète la tendance à l'affirmation d'une personnalité distincte. Il affirme l'opposition entre la pensée fédérale et les initiatives québécoises en matière de patrimoine, soulignant la contradiction identitaire entre le Canada et le Québec, dénonçant la «provincialisation» de la culture québécoise et réclamant la responsabilité sans partage du Québec dans la culture. 
Ce document pose le patrimoine comme partie prenante d'un environnement culturel plus large, formalisant ainsi la tendance amorcée dans les années précédentes. Les politiques qui suivront sa publication seront axées sur la participation accrue d'autres intervenants, comme les municipalités et les communautés locales, sur la régionalisation et sur la valorisation des activités de diffusion par le biais des musées et des centres d'interprétation ${ }^{11}$.

Pour la période 1965-1985, la présence québécoise dans les lieux historiques de la région de Québec évolue par tâtonnements, mais tend vers l'affirmation d'un patrimoine distinct; les lieux historiques sont de plus en plus considérés comme des foyers de développement identitaire, et l'accent est progressivement mis, au milieu des années 1970, sur l'interaction avec les communautés locales. La présence fédérale, par contre, est pensée en termes stratégiques, sur la base d'une idée canadienne de l'identité, de la supériorité de cette idée (et de son patrimoine) sur les autres, ainsi que d'une large diffusion publique; la gestion patrimoniale reste cependant une chasse jalousement gardée des fonctionnaires.

Quelles que soient les contradictions entre les deux paliers de gouvernement, il se dégage toutefois une vision commune sur plusieurs points. Tout d'abord, le rôle du patrimoine est renversé. On l'apprécie moins pour l'ambiance qu'il dégage que pour les leçons d'histoire qu'il permet de donner au public.

11. Pour comprendre l'évolution des politiques québécoises en patrimoine, voir notamment: Gouvernement du Québec, Commission royale d'enquête sur les problèmes constitutionnels (Québec, 1956), en particulier le vol. II: «La province de Québec et le cas canadienfrancais» et le vol. III, tome 1, «Analyse des besoins et recommandations»; Jean Lesage, «Discours prononcé à l'Assemblée législative lors de la présentation du projet de loi relatif à la création du ministère des Affaires culturelles, le 2 mars 1961 (texte intégral)», Giuseppe Turi, dir., Les problèmes culturels du Québec (Montréal, Éditions La Presse, 1974), 18-31; les Rapports annuels du ministère des Affaires culturelles de 1961-1962 à 1984-1985; Gouvernement du Québec, Livre blanc (novembre 1965) (Québec, Ministère des Affaires culturelles, 1976); Jean-Paul L'Allier, Pour l'évolution de la politique culturelle (Québec, Ministère des Affaires culturelles, 1976); Ministère d'État au Développement culturel, La politique québécoise de développement culturel (Québec, Éditeur officiel, 1978), 2 tomes; Colloque Place Royale, 1617-18 novembre 1978: Actes du Colloque (Ministère des Affaires culturelles, 1979); Gouvernement du Québec, La loi sur les biens culturels et son application (Québec, Ministère des Affaires culturelles, 1987). Pour une analyse approfondie de ces politiques, voir, entre autres: Paul Bélanger et Pierre Paquet, «La crise culturelle de la société québécoise», Claude Ryan, dir., Le Québec qui se fait (Montréal, Hurtubise HMH, 1971), 87-95; Michel Audet, "La quête d'un État: la politique québécoise de développement culturel», Recherches sociographiques, 20,2 (mai-août 1979): 263-275; Paul-Louis Martin, «La conservation des biens culturels, 65 ans d'action officielle», Rapport annuel 1986-1987 (Québec, Commission des biens culturels, 1987), 17-33; Richard Handler, Nationalism and the Politics of Culture in Quebec (Madison, University of Wisconsin Press, 1988); Paul-André Linteau, René Durocher, Jean-Claude Robert et François Ricard, Histoire du Québec contemporain, tome II: Le Québec depuis 1930 (Montréal, Boréal, 1989). 
Dans le même processus, les contenus sont orientés vers une image radicalement différente de l'identité québécoise. L'idée de modernité traverse toute l'interprétation du passé. Il est remarquable que cette lecture de l'histoire touche tous les lieux administrés par l'État, quel que soit le palier de gouvernement, ainsi que les lieux mixtes, alors qu'elle tarde à se manifester dans les lieux institutionnels.

\section{3 - CONTENU DES LIEUX HISTORIQUES}

Examinons maintenant le discours diffusé par les lieux historiques en fonction des trois axes de l'affirmation identitaire que sont l'émergence d'un être moderne en rupture avec la tradition, le rapport entre le Nous et l'Autre et le rôle du patrimoine dans la mémoire.

\section{A - Modernité et tradition}

Lorsque nous avons cherché à savoir comment s'articulait le discours sur la modernité et la tradition, nous avons d'abord constaté que ces notions ne sont jamais traitées de manière explicite. Ce ne sont pas des enjeux avoués de la mémoire même si, dans le discours, leur opposition apparaît constamment sous de nombreux déguisements. Par ailleurs, nous avons abordé la question en considérant les lieux historiques des années 1965-1985 comme des reconstitutions conformes à la grande idée du «Québec moderne», c'est-à-dire présentant un passé réadapté en fonction des aspirations nouvelles.

Les lieux occupés par les institutions religieuses et militaires, comme l'Hôpital général ou la Citadelle, n'ont pas été atteints par ce nouveau regard au même titre que les autres. Se voulant davantage le miroir de l'évolution de leur communauté que de la société, ils expriment une continuité qui abolit presque toute distance entre le passé et le présent. La mémoire des acteurs historiques, les fondateurs de ces institutions, par exemple, vit aujourd'hui avec la même intensité qu'autrefois. Si la modernité est évoquée, c'est sous la forme d'adaptations techniques qui facilitent l'accomplissement, encore une fois, de la fonction traditionnelle de l'institution (les appareils nécessaires aux soins des malades, par exemple, ou l'armement dans le cas de la Citadelle). Il faut souligner que les lieux occupés par ces institutions ont conservé leur fonction originale d'hôpitaux ou de base militaire.

Partout ailleurs, au contraire, la rupture entre le passé et le présent prend d'abord la forme d'une nouvelle affectation du lieu, transformé en musée ou en centre d'interprétation. La clientèle n'est pas appelée à regarder cet environnement avec les mêmes yeux. Si tous les lieux 
historiques jouent en partie sur la valeur nostalgique ou pittoresque des objets et des bâtiments, l'affectivité du visiteur est davantage sollicitée dans les musées institutionnels et privés, où il reçoit peu d'explications. Dans les lieux modernes d'interprétation, il est plus encadré et entraîné dans une didactique du passé. On transmet par cette pédagogie une tradition inventée où le passé est revu et corrigé en conformité avec les valeurs du jour.

Les notions de modernité et de tradition sont exprimées de manière plus explicite dans certains thèmes historiques, par exemple la présence amérindienne ou le régime seigneurial.

Ainsi, dans tous les lieux que nous avons visités, y compris à Wendake, les Amérindiens sont présentés comme des êtres de tradition. Dans quelques musées, on expose même des objets amérindiens contemporains, comme des canots d'écorce en modèle réduit ou des poupées habillées en tuniques à franges, en les présentant pêlemêle avec des objets anciens sans distinguer les époques, comme si les cultures amérindiennes échappaient à toute évolution.

Quatre lieux parlent du régime seigneurial. Au Manoir MauvideGenest et au Moulin de Beaumont, qui sont des lieux privés établis avant 1960 et où l'on conserve une collection hétéroclite de souvenirs, le régime seigneurial apparaît comme une époque révolue mais chargée de la nostalgie du «bon vieux temps». Par contre, à la Seigneurie des Aulnaies et au Domaine Joly de Lotbinière, sites ouverts au cours des années 1970-1980, le seigneur en titre est un personnage qui «modernise» sa propriété en installant un outillage neuf au moulin, en meublant son manoir au goût du jour ou encore en plantant des arbres aux essences nouvelles. Ainsi présenté, le seigneur est donc luimême porteur de modernité, ce qui rompt avec l'image habituelle d'un régime seigneurial faisant obstacle au progrès.

À Québec même, dans tous les lieux historiques implantés par l'État entre 1970 et 1984, c'est-à-dire à Place-Royale et dans les propriétés de Parcs Canada, les vecteurs de la modernité sont le marchand et l'activité portuaire, de la Nouvelle-France à aujourd'hui. L'industrie et le commerce jouent un rôle historique déterminant, qui remplace celui des institutions et des seigneurs. Evidemment, cette présentation du passé suit de très près la relecture historiographique des années 1960. Nous avons noté par ailleurs que l'État n'est lui-même jamais présenté comme une force motrice. On préfère souligner le rôle de certains de ses mandataires, comme Jacques Cartier, Samuel de Champlain et Jean Talon, qu'on associe à ces qualités «modernes» que sont l'esprit d'entreprise ou la clairvoyance commerciale. 
B - «Nous» et l'Autre

Lorsque nous avons cherché à repérer, dans le propos des lieux historiques, les couples du Nous et de l'Autre, fondements mêmes de l'identité, plusieurs pôles ont émergé sur la base de la religion, de l'appartenance ethnique ou raciale, et du rôle social. On constate d'ailleurs souvent, dans un même lieu, la présence de plusieurs couples du Nous et de l'Autre, qui correspondent à divers niveaux d'appartenance possibles.

Dans la période 1950-1960, on observe partout l'affirmation d'une identité fondée sur la foi chrétienne, mais celle-ci disparaît du discours dans la décennie 1970. Par la suite, on insiste sur des intérêts et des symboles nouveaux, en particulier sur l'esprit commercial, l'innovation et la modernité.

Dans l'ensemble, l'identité nationale (québécoise ou canadienne) est toujours implicite. Nous n'avons noté que deux exceptions: la Citadelle et le Fort-Numéro-Un, où l'identité canadienne est affirmée avec force et associée aux activités militaires. Il faut cependant se rappeler que, dans les lieux fédéraux, une plaque commémorative est chargée de rappeler le rapport du lieu à l'édification du Canada.

Dans les lieux militaires, on invite naturellement les visiteurs à adhérer, ne serait-ce que pour quelques minutes, à une vision martiale du monde. On ne questionne pas le rôle de l'armée, et on relègue systématiquement à l'arrière-plan les rivalités entre Français et Anglais. On présente même l'occupation de ces lieux, par exemple le Parc de l'Artillerie ou les Fortifications de Québec, comme une continuité sans conflit, ce qui est étonnant dans une ville qui a connu quatre sièges et trois changements d'allégeance!

Les Amérindiens sont invariablement présentés comme des êtres de tradition et une masse indifférenciée et homogène dans l'espace et dans le temps. À Place-Royale, par exemple, lorsqu'on évoque leur histoire, le seul «événement» digne de ce nom pour eux est l'arrivée des Européens, comme s'ils n'avaient pas eux-mêmes d'autonomie historique.

Nous avons également noté que la place des femmes n'est jamais explicitement soulevée, même dans les communautés religieuses de femmes. Seules les Ursulines l'abordent, mais strictement en rapport avec leur mission éducatrice. Les femmes sont souvent présentes, mais on n'évoque généralement leur rôle que par le biais de la famille: le lieu historique est d'abord un monde d'hommes.

Si les clivages sociaux sont plus volontiers explicités, ils le sont dans des lieux consacrés à des activités économiques, comme au 
Vieux-Port ou au Parc de l'Artillerie (qui abrite une ancienne fabrique de munitions), rarement ailleurs (le Fort-Numéro-Un, dont la construction est largement décrite, est une exception notable). Les conflits sociaux, par contre, sont largement ignorés.

\section{$C$ - Le rôle du patrimoine dans la mémoire}

Nous avons enfin cherché à savoir si les lieux historiques présentent un regard critique sur le passé, ou encore si les événements du passé remettent en question certains aspects du présent. Nous avons constaté que non. En général, le passé est un monde homogène, ordonné, aux relations harmonieuses, tel que le décrit le Livre blanc de 1978:

Les Québécois de tradition française ont gardé [...] une base d'unité qui, depuis trois siècles, leur a évité les déchirements et les divisions des guerres civiles ou des unifications plus ou moins forcées. Ils ont ainsi un passé collectif qui n'a pas dressé entre eux des obstacles émotifs ou idéologiques insurmontables ou infligé des blessures sociales comparables à ce que bien d'autres peuples occidentaux ont vécu. [...] Cette homogénéité de base n'a pas empêché la variété des coutumes et des images culturelles. (p. 58)

Il arrive que les conflits soient évoqués, mais on ne les attribue pas aux fondements de notre société, comme s'ils n'étaient que l'expression de déficiences corrigibles. Par ailleurs, le passé est présenté sous la forme d'un discours fini, ne laissant aucune place au débat ou aux contestations, ce qui confine le visiteur dans un rôle passif, et renforce le caractère totalitaire de cette forme de mémoire publique.

Néanmoins, nous avons noté une évolution du discours patrimonial pendant la période étudiée. Au cours des années 1950-1960, les lieux historiques, encore peu nombreux, parlent de la pérennité des institutions traditionnelles que sont l'Église et l'armée. Au cours des années 1970-1980, on insiste au contraire sur les activités commerciales et industrielles. On passe alors de la commémoration à l'interprétation, de la collection nostalgique à la collection explicative, d'un discours axé sur la contemplation à un discours didactique.

La perception du patrimoine suit la même évolution. Dans les lieux les plus anciens, le patrimoine consiste en de beaux objets ou des bâtiments qui ont "vécu», et dont la fonction est de montrer la richesse et l'ancienneté de la communauté ou de l'élite sociale. Dans les sites les plus récents, il prend la forme d'un décor reconstitué, où le discours tenu sur les bâtiments eux-mêmes, et notamment sur le fait 
qu'ils sont des reconstructions hypothétiques, est relégué au second plan. Dans les formes les plus poussées et les plus coûteuses de mise en valeur que sont Place-Royale et le Parc-de-l'Artillerie, l'histoire est ainsi transformée en un monde exotique et un spectacle pour touristes.

\section{QUELQUES CONCLUSIONS GÉNÉRALES}

Notre recherche représente un premier déblayage dans ce champ encore peu étudié de la mémoire publique qu'est le lieu historique. Les conclusions que nous en tirons mériteraient donc d'être approfondies.

\section{A - Évolution des lieux historiques}

Dans un premier temps, de 1960 à 1969, les lieux historiques sont essentiellement des lieux de mémoire institutionnelle et privée, tournés vers une communauté restreinte. Le début des années 1970 est au contraire marqué par des investissements massifs de l'État dans la mémoire, et par l'ouverture de sites destinés au grand public. Cet effort est partagé par les deux paliers de gouvernement, provincial et fédéral. Sur le plan de l'investissement, cependant, le fédéral domine nettement, en particulier à Québec même. Au milieu de la décennie 1970 commencent à émerger des lieux semi-publics qui répondent à la fois aux pressions des milieux patrimoniaux et à la redéfinition des politiques culturelles du gouvernement du Québec dans un contexte de rivalité avec celui du Canada.

\section{B - Contenu des lieux historiques}

Au début de la période étudiée, on sent encore la marque de la puissance des grandes institutions religieuses, mais avec les années 1970, l'État prend le relais. Dans tous les cas, le contenu des lieux d'interprétation est marqué par l'absence de critique sociale et politique et une glorification implicite des élites (seigneurs, personnages religieux et militaires, marchands).

Si nous revenons aux hypothèses générales du début, nous avons constaté que les lieux historiques confirment, par leur discours et par la rapidité de leur émergence, la naissance symbolique du Québécois moderne en rupture avec son passé.

D'autre part, c'est l'État, autoproclamé comme l'incarnation de la volonté et du bien communs, qui prend en charge cette reconfiguration. Aussi peut-on dire que, malgré son absence apparente du discours des lieux historiques, l'État est omniprésent comme instance énonciatrice. Le patrimoine, mis en vitrine, catalogué et aseptisé, se présente de la sorte comme inoffensif et malléable. 
Nous sommes bien loin d'avoir épuisé le sujet. Nous estimons, au contraire, que le statut de l'histoire diffusée par les lieux historiques offre un terrain d'enquête fertile où seraient mises à contribution l'histoire, l'ethnologie, la muséologie et la sociologie. Si les lieux historiques sont le reflet d'un certain regard de la société québécoise sur elle-même, ils sont aussi les agents d'une large diffusion du discours sur le passé. Cela nous amène à insister sur l'idée que les lieux patrimoniaux ne doivent pas échapper au regard critique de la recherche historique. 